\title{
The Establishment of Young Women Jogging Pants Suit Dynamic Type Version Based on Three- dimensional Human Body Data
}

\author{
Xian $\mathrm{Li}^{1}$ and Minghai Cui ${ }^{2, *}$ \\ Beijing Institute of Fashion Technology, Yinghuadongjie, Beijing, 100029, China \\ ${ }^{*}$ Corresponding author
}

\begin{abstract}
Through three-dimensional scanning technology, the dynamic human body data of young women jogging is obtained. This paper applies Auto CAD plug in, and aims at the wire-frame model of scanning action to carry out a experiment which transforms three dimensions into two dimensions. The sample plate obtained through this method can reflect the leg shape of human body directly. After combining with dynamic sample plate when it flats running, the adjusted pattern can be taken as an important basis for running pants pattern design directly.
\end{abstract}

Keywords-three-dimensional scanning; young women; jogging pants; version optimization

\section{PREFACE}

Recently, people prefers to the jogging because of its advantages of simpleness and easiness to do. More and more young people choose jogging in order to keep figure and fit. In addition, with the development of science and society, people need more qualified and delicate sports clothes. Therefore, it is extremely important to analyze the structure of jogging clothes, and design the beloved, beautiful and comfortable sports clothes.

This paper starts from the body movement, analyzes the changing conditions of human leg skin when they are dynamic through the dynamic scanning of 3-D scanning, makes the flat experiments through the human body data, and provides the referential according for the improving design of the young female jogger pants style.

\section{THREE DIMENSIONS BODY SCANNING EXPERIMENT}

\section{A. Experimental Object}

The experimental objects of this study are ten undergraduates and postgraduates in a university in Beijing. Their ages are between 18 and 28, and their BMI is from 19 to 23.

\section{B. Experimental Facilities and Environment}

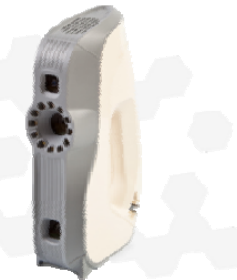

FIGURE I. ARTEC EVA RAPID HANDLE 3D SCANNER

The experimental environmental temperature is $26 \pm 2^{\circ} \mathrm{C}$; the relative wet is $(60 \pm 10) \%$; the facility is Artec Eva Rapid Handle 3D Scanner; a computer station with the Artec Eva Rapid Handle 3D Scanner software; two sets of Martin's meter; two sets of horizontal marker and holder; measuring units and so on. The establishment of experimental scanning postures.

\section{The Establishment Of Experimental Scanning Postures}

This paper mainly uses the measuring method of the combination of direct measurement and indirect measurement. The direct measurement is the Martin and tape measurement; and the indirect measurement is the 3-D scanner. The experimental movements mainly include join standing and split standing, and jogging movements including forward swing, backward pedal and landing buffer. Among these: (1)the forward swing stage is the start stage of jogging; at this time, the swing leg and the horizontal level form about 30 degree; the joints of the standing leg and the vertical level form about 20 degree. (2)At the landing buffer stage, the raised leg rapidly lands to enter the backward pedal stage, as showed in Figure II. The concrete movement standard is shown in Figure III.

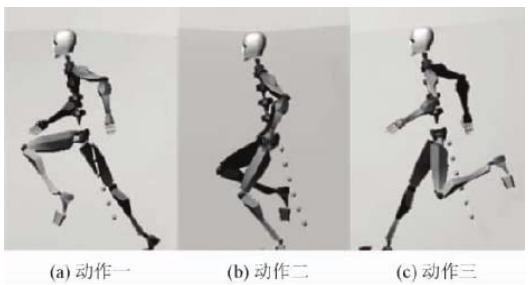

FIGURE II. DECOMPOSITION OF JOGGING MOVEMENTS 


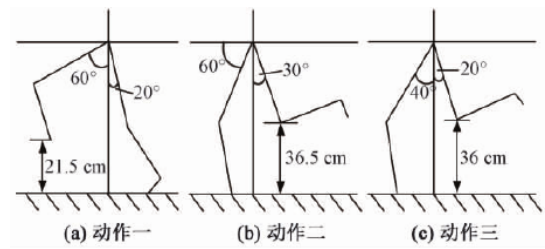

FIGURE III. LINE PRESENTING FIGURE

\section{Experimental Process}

Stick the measuring point --- draw the measuring lines (horizontal and vertical line) -- measure by hand (this experiment chooses 102 Martin measurement projects, which are measured by the professional people with the equipped measuring machine and write down the data. Among these, the measuring objects relating to the lower body are 60.) -- 3D scanning data (use Artec Eva 3D handle scanning machine to collect the 3D body data to the five postures of the volunteers).

\section{DYNAMIC DATA ANALYSIS}

\section{A. Data Choosing and Dealing}

Put the original experiment data into the software Artec Studio 9, through the dealing of data edition, matching, combination, texture mapping and data export, finally obtain 50 3D body data. The leg skin stretching conditions in the start stage and backward pedal stage and in standing are different in the jogging process. Therefore, this paper make the second and third movements as the researching object. The Figure shows the data of the three split standing after exporting.

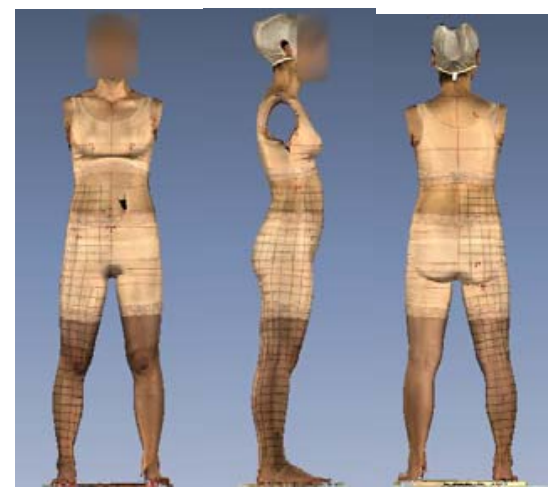

FIGURE IV. DATA AFTER BEING DEALT BY ARTEC STUDIO9

Put the data from the last stage into the software Rapid form XOR3, and will get the complete, smoothing body scanning data after taking out the spot, bypass to fill holes and other dealings. Then use the line drawing tools to draw the measuring lines in the former handle lines in the software to prepare for the analyzing the length change of the line segment. And use the line segment to cut the surface into the surface segment to prepare for the analyzing the area change, as is shown in Figure V.

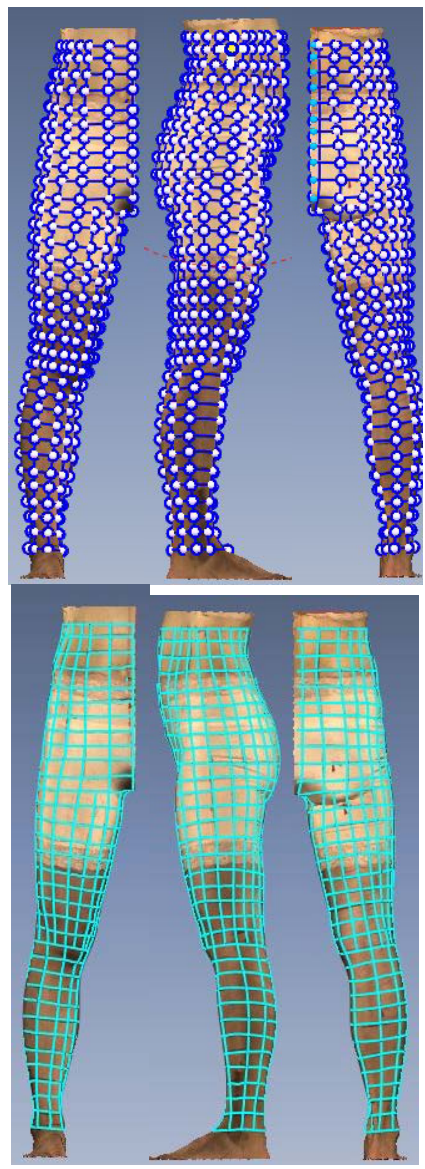

FIGURE V. DATA AFTER LINE DEALING AND SURFACE DEALING

\section{B. Data Analysis}

\section{1) Surface area analysis}

The structural line design of the jogging pants mainly relates to the skin shrinking condition of the lower leg under the sports condition, body wet and warm condition and the material kind and features. This paper mainly uses the levels in Figure to present the area changing ratio, and presents the leg parts through different section change. The calculation of the changing ratio is the following formula:

\section{Practical dynamic data - Natural standing data $* 100 \%$ Natural standing data}

Through this formula calculate the data changing ratio of every leg section in steady and dynamic conditions. The result is that the data changing ratio is positive and negative. The positive represents the skin stretching phenomenon, and the negative represents the skin shrinking. The ratio range is from $90 \%$ to $90 \%$, with less in $-80 \%$ to $80 \%$. This paper classifies 16 levels of change ratio, using different colors to represent different levels, with $10 \%$ as a level gap every two colors. The average distribution of the surface area change ratio of the ten measured joggers is shown in Figure VI. 


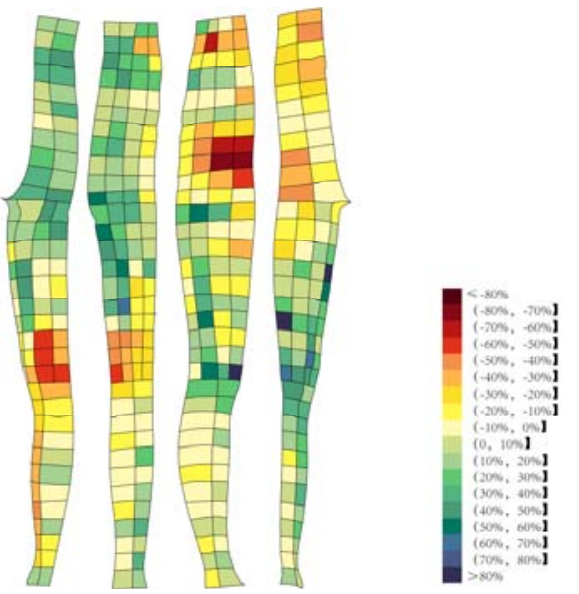

FIGURE VI. LEVEL FIGURE OF LEG AREA CHANGE RATIO

The level Figure of surface area change ration presents the following regular: in the condition of the jogging movement 1 , the shrinking ration in the front waist and abdomen part is big, below $-20 \%$, especially the middle part of groin and knee present the shrinking ratio below $-50 \%$; the skin area stretching variation on the back waist, hip, the upper part of the back leg and the knee is big, between $10 \%$ to $30 \%$, among which the changing on top hip, hip gap and the middle part of knee is over $50 \%$; the stretching amount on the back leg near the inner gap is relative big, between $20 \%$ and $40 \%$; the skin stretching ratio on the lower middle part of the front leg is between 0 to $20 \%$; the skin stretching ratio on the ankle is between 0 to $20 \%$.

\section{2) Flat spread of the surface data}

Choose a middle Figure from the ten body scanning dynamic in the former measurement, apply the Auto CAD plug-in, make the right line model of the split standing movement become the planer triangle network. The dynamic basic model through this method can directly reflect leg structure. The pattern adjusted from the planer movements 3 and 5 can be used as the important accordance of the jogging pants pattern design.

In the planer process, the former hip surrounded line and thigh line keep horizontal and keep vertical with the former returning line. The back thigh line keeps horizontal and vertical with the back returning line. The back hip surrounding line raises according to the hip top amount. Through the method of further cutting spreading, movement and turning, it will get the basic pants pattern striped from human body. The stripping pattern exists a certain of repetition on the fork bending radian, which is the unplug amount of the fork bending radian and can be dealt through techniques. The unfolding result is shown in Figure VII.

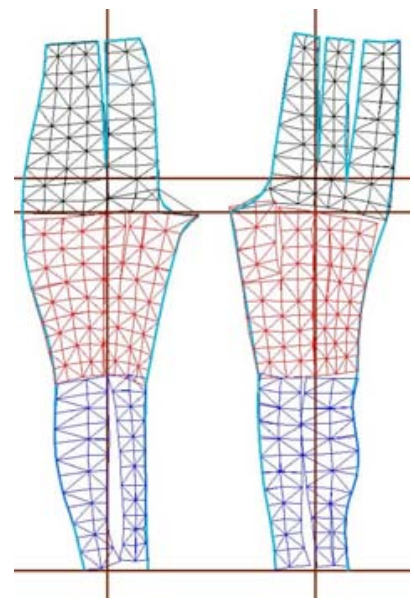

FIGURE VII. DYNAMIC LEG PLANER UNFOLDING FIGURE

After obtaining the dynamic basic pattern, do the planner unfolding experiment of the transformation from 3D to planner to the first and second jogging movements, leading to the jogging original dynamic pattern showed in Figure VIII and Figure X.

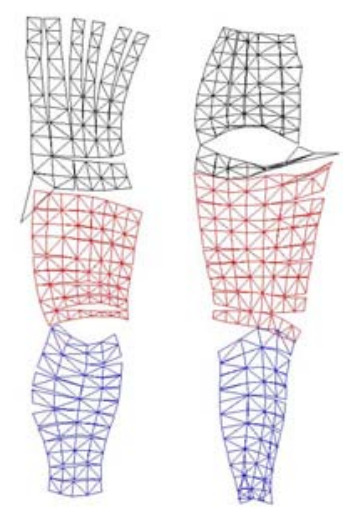

FIGURE VIII. ORIGINAL MOVEMENT PATTERN OF THE THIRD MOVEMENT

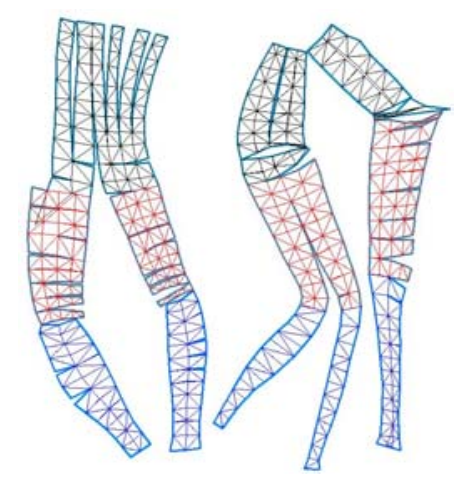

FIGURE IX. ORIGINAL MOVEMENT PATTERN OF THE THIRD MOVEMENT AFTER SETTLEMENT

From the unfolding result, we can see that when people are jogging, the big change of skin size mainly focuses on the back hip part, the front and back leg, the front and back knee and the back shank. 
Settle the dynamic pattern from the result of 3D to planer. Make the save-amount transformation. After jointing, combing and adjusting, we will get the dynamic pattern cut-off rule shown in Figure IX and Figure X. The pattern adjustment is accorded by the transforming position of the dynamic pattern on the human body. The cut-off line dividing in this method more accords to the moving body, and these line design also concerns partly functional design.

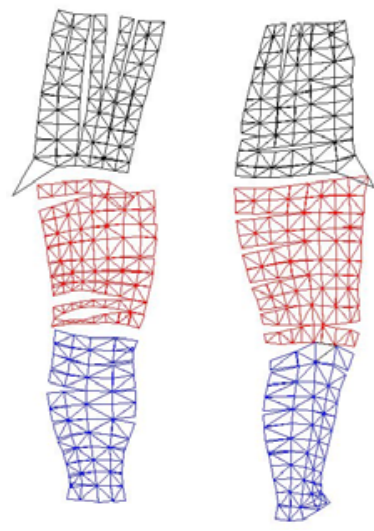

FIGURE X. ORIGINAL DYNAMIC PATTERN OF THE FIFTH MOVEMENT

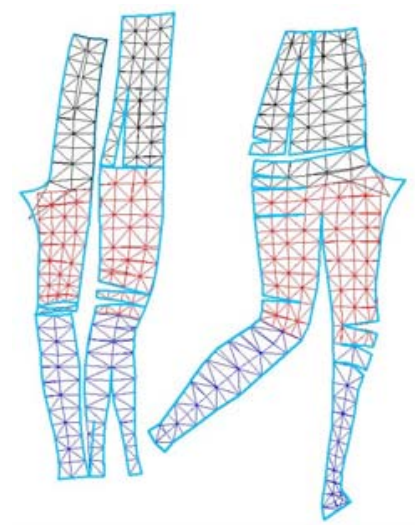

FIGURE XI. DYNAMIC PATTERN OF SETTLED FIFTH MOVEMENT

According to the surface line segment stretching variation result of the lower key parts under the condition of jogging in the first period, we can know that we should think over the following questions when designing the jogging pants: (1)on the materials choice, we should choose fine gauze directive elastic force over the weft directive elastic materials to guarantee the vertical flexible possibility of the skin and muscle; (2) the bigger changing ratio parts when jogging are: hip, former, outside and back leg should be fully considered the stretching space. The big changing ration parts such as the leg root and horizontal crotch should be given enough stretching space. In these regions, it should be considered the comfort and beauty. (3) according to the moving feature when jogging, the back rise should have more space than the former rise to ensure the comfort of sports. (4) knees. (5) waist and abdomen: the part under knees needn't much loose design. When choosing the jogging pant materials, we can choose the materials less elastic.

\section{CONCLUSION}

In the pattern improving process of the jogging pants, through the data from 3D scanning experiment, it should take the method of the dynamic and steady combination when designing $t$ he pattern of jogging pants. Only researching the actual moving process and analyzing the surface data during the sports process can we design the jogging pants pattern structure which comfort to body and sports comfort.

\section{REFERENCES}

[1] Yan-zhen Wang, Jian-ping Wang, Yan Zang, Ting-ya Wu. Study on Skin Tension Based on Running Condition [J]. Journal of Textile Research.2013,34(8)

[2] [Japan] Miyoshi Machiko. Clothing modeling theory articles[M].Zheng Rong, translation. Beijing: China Textile Board.2006:67 84.

[3] Liu Wei. Study on the Structure Design of Men's Cycling Pants Based on Motion Analysis [D]. Beijing: Beijing Institute Of Fashion Technology.2013.

[4] Dan Cao. Study on Optimized Design of Functional Running Pants Structure[D].Beijing: Beijing Institute Of Fashion Technology.2015. 